\title{
Supersymmetric dark matter Q-balls and their interactions in matter
}

\author{
Alexander Kusenko ${ }^{1,2}$, Lee C. Loveridge ${ }^{1}$, and Mikhail Shaposhnikov ${ }^{3}$ \\ ${ }^{1}$ Department of Physics and Astronomy, UCLA, Los Angeles, CA 90095-1547 \\ ${ }^{2}$ RIKEN BNL Research Center, Brookhaven National Laboratory, Upton, NY 11973 \\ ${ }^{3}$ Institute of Theoretical Physics, Swiss Federal Institute of Technology (Lausanne), BSP, CH-1015 Lausanne, Switzerland
}

\begin{abstract}
Supersymmetric extensions of the Standard Model contain non-topological solitons, Q-balls, which can be stable and can be a form of cosmological dark matter. Understanding the interaction of SUSY Q-balls with matter fermions is important for both astrophysical limits and laboratory searches for these dark matter candidates. We show that a baryon scattering off a baryonic SUSY Q-ball can convert into its antiparticle with a high probability, while the baryon number of the Q-ball is increased by two units. For a SUSY Q-ball interacting with matter, this process dominates over those previously discussed in the literature.
\end{abstract}

PACS numbers: 11.30.Pb 12.60.Jv 95.35.+d 14.80.Ly

\section{INTRODUCTION}

Supersymmetry (SUSY) is widely regarded as a plausible candidate for physics beyond the Standard Model. In addition to its aesthetic appeal and its ability to stabilize the hierarchy of scales, supersymmetry provides dark matter candidates in the form of the lightest supersymmetric particles and SUSY Q-balls.

Q-balls are nontopological solitons that carry some conserved global quantum number [1, 2]. They exist in SUSY extensions of the standard model [3] and are either stable or have lifetimes in excess of the age of the universe in theories with gauge-mediated SUSY breaking [4]. Stable SUSY Q-balls have large baryon numbers; the vacuum expectation value (VEV) of the scalar field inside these Q-balls lies along some "flat direction" of the effective potential.

Cosmological evolution of SUSY scalar fields after inflation can give rise to both ordinary matter, via AffleckDine baryogenesis [5, 6], and to dark matter in the universe in the form of SUSY Q-balls 7] or neutralinos from Q-ball decays [8]. Since both atomic matter and dark matter arise from the same process, it may be possible to relate their amounts in the universe $9,10,11,12,13$. In theories with gravity mediated SUSY breaking, all Q-balls are short-lived, but their production can change the standard way of computing neutralino abundances [10, 11, 12, 14].

If baryonic SUSY Q-balls make up the dark matter in the universe, they may be detected [15] in current or future experiments, such as ANTARES, Baikal, IceCube, Super-Kamiokande, etc. A number of bounds on Q-balls already exist in the literature [15, 16].

Specific experimental signatures, which could be used to detect SUSY Q-balls and to distinguish them from other heavy dark-matter candidates, such as strangelets, nuclearites, and monopoles, depend on the Q-ball interactions in matter. These interactions also determine the astrophysical bounds, which will be discussed elsewhere [17]. Baryonic Q-balls in gauge-mediated scenarios have mass per baryon below $1 \mathrm{GeV}$. Hence, storing baryon number in Q-balls is energetically favored over nucleons. It is clear that a nucleon can be absorbed by a Q-ball and the energy difference can be released. However, the rates of such interactions have not been computed accurately.

It was conjectured in Ref. 15 that an absorption of a nucleon by a Q-ball occurred essentially in two stages. Since the color SU(3) symmetry inside a baryonic SUSY Q-ball is broken [18, it was concluded that the first stage is a nucleon disintegration into quarks, in which the energy released (in pions) is of the order of the binding energy of quarks, that is $\sim 1 \mathrm{GeV}$. The next step was a decay of quarks into the scalar condensate inside the Q-ball. This process is, of course, allowed because the energy per baryon number in the condensate is small. The rate of this second process was estimated to be suppressed by a factor $(E / \Lambda)^{3}$, where $\Lambda \sim 1 \mathrm{TeV}$ is the scale of SUSY breaking and $E \sim 1 \mathrm{GeV}$ is the typical quark energy. This estimate was essential for the discussion of Q-balls interactions in a neutron star [19]. On the basis of this estimate it was concluded in Ref. [19] that a neutron star can survive for at least 1 Gyr after capturing a dark-matter Q-ball. We will see that the picture of interaction of baryons with Q-balls discussed above is incorrect.

The goal of the present paper is to reanalyze the interaction of Q-balls with ordinary matter. We find, in fact, that quarks, falling on a Q-ball, are reflected as antiquarks with a probability on the order of one, practically independent of the parameters of the theory. In other words, Q-balls convert the matter into antimatter on their surface (or antimatter into matter, if placed in an anti-matter environment). Baryon number is conserved during this process: after reflection of an antinucleon, the baryonic charge of the Q-ball increases by 2 units. 


\section{INTERACTIONS OF QUARKS WITH SUSY Q-BALLS}

\section{A. SUSY Q-ball basics}

Let us consider some flat direction of a supersymmetric extension of the standard model. This flat direction can be parameterized by a scalar field $\varphi$. The VEVs of squarks and sleptons are proportional to $\varphi$ along the flat direction. In theories with gauge mediation the effective potential along this flat direction practically does not grow $V(\varphi) \propto \Lambda^{4}$, up to some $\varphi_{\max }$ which depends on the flat direction [23]. There exists a classical spherically symmetric solution of the field equations, a nontopological soliton, in which

$$
\varphi=\phi_{0} f(r) e^{i \omega t}
$$

where $f(r) \simeq \sin (\omega r) /(\omega r)$ for $r \leq R=\pi / \omega$ and zero for $r>R$. It carries the global charge $Q$. If $\phi_{0}<\varphi_{\max }$, different parameters depend on $Q$ as follows:

$$
\phi_{0} \sim \Lambda Q^{1 / 4}, \quad M \sim \Lambda Q^{3 / 4}, \quad \omega \sim \Lambda Q^{-1 / 4}
$$

\section{B. Structure of the quark mass term inside Q-ball}

For us the relevant parts of the MSSM Lagrangian are those which describes interactions of quarks $\psi$ with squarks $\phi$ and gluinos $\lambda$ :

$$
\mathcal{L}=-g \sqrt{2} T_{i j}^{a}\left(\lambda^{a} \sigma^{2} \psi_{j} \phi_{i}^{*}\right)+C . C .+\ldots
$$

and also the Majorana mass terms for gluinos:

$$
\mathcal{L}_{\mathcal{M}}=M \lambda_{a} \lambda_{a}
$$

In theories with gauge mediation the Majorana gluino mass stays constant along the flat directions of the effective potential [22].

Inside a Q-ball the squarks have a non-zero expectation value $\langle\phi\rangle=\varphi$, and, therefore, the quarks acquire a non-zero mass through their mixing (3) with gluinos. In general, the mass matrix has many terms of the form

$$
M_{i}^{a} \lambda^{a} \sigma^{2} \psi_{i}
$$

where

$$
M_{i}^{a}=-g \sqrt{2} T_{j i}^{a} \varphi_{j}^{*} .
$$

To describe the interactions of quarks with the Q-ball, it is sufficient to consider a simplified mixing matrix

$$
\left(\begin{array}{ccc}
0 & m & \varphi_{L} \\
m & 0 & \varphi_{R} \\
\varphi_{L} & \varphi_{R} & M
\end{array}\right)
$$

where $\varphi_{L, R}$ represent the mixing terms for the left and right handed components of the quark field, and are proportional to the left and right handed squark expectation values. The factor of $-\sqrt{2} g$ has also been absorbed into the definition of these parameters. Since $\omega \ll \phi_{0}$, we neglect the time dependence of the Q-ball solution for now. It will be addressed below. As a further simplification, one can neglect the quark Dirac mass $m$, since along the MSSM flat directions the Higgs field is equal to zero [21]. We parameterize the left and right handed mixing terms as

$$
\left(\begin{array}{c}
\varphi_{L} \\
\varphi_{R}
\end{array}\right)=\varphi\left(\begin{array}{c}
\cos \alpha \\
\sin \alpha
\end{array}\right)
$$

Then one linear combination of left and right handed quark fields

$$
\left(\begin{array}{c}
\chi_{L} \\
\chi_{R}
\end{array}\right)=\chi_{\text {massless }}\left(\begin{array}{c}
\sin \alpha \\
-\cos \alpha
\end{array}\right)
$$

remains massless. The other, massive component

$$
\left(\begin{array}{l}
\chi_{L} \\
\chi_{R}
\end{array}\right)=\chi_{\operatorname{mass}}\left(\begin{array}{c}
\cos \alpha \\
\sin \alpha
\end{array}\right)
$$

is mixed with the gluino according to a mass matrix of the form

$$
\left(\begin{array}{cc}
0 & \varphi \\
\varphi & M
\end{array}\right)
$$

where $\varphi=\sqrt{\varphi_{L}^{2}+\varphi_{R}^{2}}$. This mass matrix can be written as

$$
\tilde{M}\left(\begin{array}{cc}
0 & \sin 2 \beta \\
\sin 2 \beta & 2 \cos 2 \beta
\end{array}\right) .
$$

It has the eigenvalues

$$
M_{+}=2 \tilde{M} \cos ^{2} \beta, \text { and }-M_{-}=-2 \tilde{M} \sin ^{2} \beta
$$

corresponding to eigenvectors

$$
\left(\begin{array}{c}
\sin \beta \\
\cos \beta
\end{array}\right) \text { and }\left(\begin{array}{c}
\cos \beta \\
-\sin \beta
\end{array}\right) \text {. }
$$

These eigenstates form Majorana particles with masses $M_{+},\left(-M_{-}\right)$.

\section{Quark scattering on Q-balls}

Let us consider the quark scattering on a Q-ball. The Q-ball size is in general much larger than the wavelength of the quarks, so it is sufficient to treat the problem as reflection from a flat boundary surface. To simplify the analysis further, we will assume that the profile of the scalar field has a $\theta$-function behavior, which does not lead to any loss of accuracy since the energy of the incident quark is much smaller than the masses $M_{+}, M_{-}$. 


\begin{tabular}{|c|c|c|c|}
\hline Particle & Description & Mass & Direction \\
\hline$A$ & Incoming Quark & 0 & + \\
\hline$B$ & Reflected Quark & 0 & - \\
\hline$C$ & Reflected Gluino & $M$ & - \\
\hline$D$ & Transmitted State & $+M_{+}$ & + \\
\hline$F$ & Transmitted State & $-M_{-}$ & + \\
\hline
\end{tabular}

TABLE I: Different states participating in the scattering problem.

Then this is a simple quantum-mechanical problem in which one has to match the the wave functions of the incident quark and the reflected quark/antiquark (and, potentially, the gluino, if the energy of the incoming quark is large enough) with the wave-functions of the Majorana fermions inside the Q-ball.

Majorana fermions obey the differential equation

$$
i \bar{\sigma} \cdot \partial \chi+i m \sigma^{2} \chi^{*}=0
$$

with the solutions

$$
\begin{array}{ll}
\sqrt{\sigma \cdot p}\left(A e^{-i p \cdot x}+\sigma^{2} A^{*} e^{i p \cdot x}\right) & \text { for } m>0 \\
\sqrt{\sigma \cdot p}\left(A e^{-i p \cdot x}-\sigma^{2} A^{*} e^{i p \cdot x}\right) & \text { for } m<0 .
\end{array}
$$

Five different wave functions are involved as summarized in Table II. The combined wave functions of the incoming quark, the reflected quark, and the reflected gluino must match the internal wave functions of the internal mass eigenstates at the boundary.

The general continuity equation is

$$
\left(\begin{array}{c}
\chi_{A}+\chi_{B} \\
\chi_{C}
\end{array}\right)=\left(\begin{array}{c}
\sin \beta \\
\cos \beta
\end{array}\right) \chi_{D}+\left(\begin{array}{c}
\cos \beta \\
-\sin \beta
\end{array}\right) \chi_{F},
$$

which simplifies to

$$
\begin{aligned}
\sin \beta \sqrt{E}(A+B)+\cos \beta \sqrt{E+M} C & =\sqrt{E+M_{+}} D \\
\sin \beta \sqrt{E}\left(\sigma \cdot \hat{\mathbf{p}} \mathbf{A} A+\sigma \cdot \hat{\mathbf{p}}_{\mathbf{B}} B\right) & \\
+\cos \beta \sqrt{E-M} \sigma \cdot \hat{\mathbf{p}_{\mathbf{C}}} C & =\sqrt{E-M_{+}} \cdot \hat{\mathbf{p}}_{\mathbf{D}} D \\
\cos \beta \sqrt{E}(A+B)-\sin \beta \sqrt{E+M} C & =-\sqrt{E-M_{-}} \sigma \cdot \hat{\mathbf{p}}_{\mathbf{F}} F \\
\cos \beta \sqrt{E}\left(\sigma \cdot \hat{\mathbf{p}} \mathbf{A} A+\sigma \cdot \hat{\mathbf{p}}_{\mathbf{B}} B\right) & \\
-\sin \beta \sqrt{E-M} \sigma \cdot \hat{\mathbf{p}_{\mathbf{C}}} C & =-\sqrt{E+M_{+}} F .
\end{aligned}
$$

In general, the gluino mass and the masses inside the Q-ball are much larger than the energy of the incoming quark. In this case $B= \pm i A$ so the quark will be reflected with unit probability as it is unable to propagate in any of the other states. (The sign is determined by the sign of the gluino mass.)

More interestingly, the reflected quark has reversed frequencies compared with the incoming quark. This is because in changing the direction, the effect of the projector $\sqrt{\sigma \cdot p}$ is reversed. Thus, if the quark comes in with a positive frequency it is reflected with a negative frequency. This in turn implies that it must either change chirality or particle anti-particle identity. (A right-handed particle is described by its left-handed anti-particle, so the
Incoming Left Handed

\begin{tabular}{|c|c|c|}
\hline & Quark & Anti-Quark \\
\hline Transmitted & $\sin ^{4} \alpha$ & $\sin ^{2} \alpha \cos ^{2} \alpha$ \\
\hline Reflected & $\cos ^{2} \alpha \sin ^{2} \alpha$ & $\cos ^{4} \alpha$ \\
\hline
\end{tabular}

Total Probability to Reflect: $\cos ^{2} \alpha=\left(\varphi_{L}{ }^{2} / \varphi^{2}\right)$

Total Probability to Change Identity: $\cos ^{2} \alpha\left(\varphi_{L}^{2} / \varphi^{2}\right)$

Incoming Right Handed

\begin{tabular}{|c|c|c|}
\hline & Quark & Anti-Quark \\
\hline Transmitted & $\cos ^{4} \alpha$ & $\sin ^{2} \alpha \cos ^{2} \alpha$ \\
\hline Reflected & $\cos ^{2} \alpha \sin ^{2} \alpha$ & $\sin ^{4} \alpha$ \\
\hline
\end{tabular}

Total Probability to Reflect: $\sin ^{2} \alpha=\left(\varphi_{R}{ }^{2} / \varphi^{2}\right)$

Total Probability to Change Identity: $\sin ^{2} \alpha\left(\varphi_{R}{ }^{2} / \varphi^{2}\right)$

TABLE II: The probabilities of a quark reflection from Q-ball as either a quark or an antiquark.

frequency flip may represent a change of handedness.) Zhitnitsky found similar results in studying reflections from baryonic color superconductors [20].

The linear combination of left and right handed quarks which remains massless will propagate through the Q-ball unimpeded and experiences no frequency flip. However, because it mixes left and right handed states, it too can result in changes in handedness and particle anti-particle identity.

The exact probability of reflection, chirality change, or particle identity change depends on the chirality and the identity of the incoming particle and the left right composition of the squark condensate. These are summarized in Table II. However, in a large collection of many leftand right-handed quarks impinging on the Q-ball, on average, half will be reflected and half will be transmitted. Half will change their identity and half will retain their identity.

The above results hold as long as all of the masses $M, M_{+}, M_{-}$are larger than the energy. Oblique reflection changes the formulas so that $B=i \sigma^{3} \sigma \cdot \hat{\mathbf{p}}_{\mathbf{A}} A$, but a unitary reflection and a frequency flip are preserved. Also if the mass of one of the internal eigenstates is only slightly larger than the incoming energy, the phase of the reflected state $B$ relative to $A$ changes from $i$ to -1 , but again unit reflection and frequency flipping are preserved.

However, if the gluino mass is much larger than the mixing terms, one of the two internal masses may be smaller than the energy of the incoming quark. In this case the quark can propagate inside the Q-ball, and the reflection and frequency flip probabilities are suppressed. The reflected state is then

$$
B=-\frac{E+M_{r}-p}{E+M_{r}+p} A
$$

In the case that the gluino mass is very large, so that $M_{r}=\frac{\varphi^{2}}{M_{g}} \ll E$ we find that the reflection probability goes like $\left|\frac{\varphi^{2}}{M_{g} E}\right|^{2}$. Since less of the original state is split off by reflection, the probability of becoming an anti-particle is also suppressed. Thus, in this case the processing of 
quarks by the Q-ball will be suppressed. This case, however, is not realistic as the typical value of the gluino mass is assumed to be in the range of hundreds $\mathrm{GeV}$ whereas the value of the squark fields inside the Q-ball is much higher.

\section{Time-Dependent Effects}

Let us now consider the time dependence of the condensate. The squark condensate in a Q-ball is not static, it oscillates slowly, so that $\varphi_{L} \rightarrow \varphi_{L} e^{i \omega t}$ and $\varphi_{R} \rightarrow \varphi_{R} e^{-i \omega t}$. (The sign is different because the righthanded quark is described by its left-handed anti-particle and therefore has opposite baryonic charge.) This can be accommodated by changing equations (9) and (10) to

$$
\left(\begin{array}{l}
\chi_{L} \\
\chi_{R}
\end{array}\right)=\chi_{\text {massless }}\left(\begin{array}{c}
\sin \alpha e^{-i \omega t} \\
-\cos \alpha e^{i \omega t}
\end{array}\right)
$$

and

$$
\left(\begin{array}{l}
\chi_{L} \\
\chi_{R}
\end{array}\right)=\chi_{\operatorname{mass}}\left(\begin{array}{c}
\cos \alpha e^{-i \omega t} \\
\sin \alpha e^{i \omega t}
\end{array}\right) .
$$

Now when we project the incoming quark onto the reflected and transmitted states and then back onto standard left and right-handed states, the extra phase cancels when the particle retains its identity and reinforces when the particle changes its particle-antiparticle identity. Thus, a quark entering with energy $\mathrm{E}$ will be changed to an anti-quark with energy $E-2 \omega$. The energy that is lost represents the amount of energy required to raise the baryon number of the condensate by $(2 / 3)$, the baryon number of two quarks. Such an energy loss is to be expected because $\omega$ can be thought of as a chemical potential of the condensate.

Some final remarks are now in order. In all considerations above we were taking quarks rather than baryons, which would clearly be more appropriate at small energies. We believe, however, that the conclusion about matter-antimatter conversion remains intact in a realistic case as well, though the computation of reflection coefficients is a very difficult task as it requires confinement effects to be taken into account. Nevertheless, we expect that a nucleon can change its baryon number with a large probability on reflection off a baryonic Q-ball. Since the lagrangian has no explicit baryon number nonconservation, this means that the missing baryon number is deposited into the squark condensate.

\section{CONCLUSIONS}

We have shown that a baryon interacting with a Qball can be reflected as an antibaryon, while the baryon number of a Q-ball is increased by two units. This process, which, in general, occurs with a large probability is the main source of energy release for a Q-ball passing through matter. In a separate publication we will reexamine some astrophysical bounds on dark matter SUSY Q-balls.

Acknowledgments. The authors thank J. Morris, E.S. Phinney, and P. Tinyakov for helpful comments. The work of A.K. and L.L. were supported in part by the US Department of Energy grant DE-FG03-91ER40662, a NASA ATP grant NAG5-13399, and by a Faculty Grant from UCLA Council on Research. The work of M.S. was supported in part by the Swiss Science Foundation.
[1] G. Rosen, J. Math. Phys. 9, 996 (1968) ibid. 9, 999 (1968); R. Friedberg, T. D. Lee, A. Sirlin, Phys. Rev. D 13, 2739 (1976); S. Coleman, Nucl. Phys. B 262, 263 (1985); A. Kusenko, Phys. Lett. B 404, 285 (1997).

[2] T. D. Lee and Y. Pang, Phys. Rept. 221, 251 (1992).

[3] A. Kusenko, Phys. Lett. B 405, 108 (1997).

[4] G. Dvali, A. Kusenko, M. Shaposhnikov, Phys. Lett. B 417, 99 (1998).

[5] I. Affleck, M. Dine, Nucl. Phys. B 249, 361 (1985); M. Dine, L. Randall, S. Thomas, Phys. Rev. Lett. 75, 398 (1995); Nucl. Phys. B 458, 291 (1996); R. Allahverdi, B. A. Campbell and J. R. Ellis, Nucl. Phys. B 579, 355 (2000); A. Anisimov and M. Dine, Nucl. Phys. B 619, 729 (2001).

[6] M. Dine and A. Kusenko, Rev. Mod. Phys. 76, 1 (2004).

[7] A. Kusenko, M. Shaposhnikov, Phys. Lett. B 418, 46 (1998).

[8] K. Enqvist and A. Mazumdar, Phys. Rept. 380, 99 (2003).

[9] M. Laine and M. E. Shaposhnikov, Nucl. Phys. B 532, 376 (1998).

[10] M. Fujii and K. Hamaguchi, Phys. Lett. B 525, 143
(2002).

[11] M. Fujii and K. Hamaguchi, Phys. Rev. D 66, 083501 (2002).

[12] M. Fujii and T. Yanagida, Phys. Lett. B 542, 80 (2002).

[13] R. Banerjee and K. Jedamzik, Phys. Lett. B484, 278 (2000).

[14] K. Enqvist, J. McDonald, Phys. Lett. B 425, 309 (1998); Nucl. Phys. B 538, 321 (1999); Phys. Rev. Lett. 81, 3071 (1998); Phys. Lett. B 440, 59 (1998); Phys. Rev. Lett. 83, 2510 (1999).

[15] A. Kusenko, V. Kuzmin, M. Shaposhnikov, P. G. Tinyakov, Phys. Rev. Lett. 80, 3185 (1998).

[16] I. A. Belolaptikov et al., astro-ph/9802223 M. Ambrosio et al. [MACRO Collaboration], Eur. Phys. J. C13, 453 (2000); B. C. Choudhary [MACRO Collaboration], hep-ex/9905023 G. Giacomelli, L. Patrizii, DFUB-9830, hep-ex/0002032, 5th ICTP School on Nonaccelerator Astroparticle Physics, Trieste, Italy, 29 Jun - 10 Jul 1998; E. Aslanides et al. [ANTARES Collaboration], astro-ph/9907432

[17] A. Kusenko, L. Loveridge, and M. Shaposhnikov, in preparation. 
[18] A. Kusenko, M. Shaposhnikov, P. G. Tinyakov, Pisma Zh. Eksp. Teor. Fiz. 67, 229 (1998).

[19] A. Kusenko, M. Shaposhnikov, P. G. Tinyakov, I. I. Tkachev, Phys. Lett. B 423, 104 (1998).

[20] A. Zhitnitsky, J. Phys. G 30, S513 (2004).

[21] T. Gherghetta, C. F. Kolda and S. P. Martin, Nucl. Phys.
B 468, 37 (1996).

[22] G. F. Giudice and R. Rattazzi, Phys. Rept. 322, 419 (1999).

[23] We omit possible log factors, because they are not essential for the discussion 